\title{
Self-assembled Structures from Amphiphilic Peptides
}

\author{
Severin J. Sigg, Thomas B. Schuster, and Wolfgang P. Meier ${ }^{\star}$
}

\begin{abstract}
Nanotechnology and its applications are strongly influenced by structures self-assembled from a variety of different materials. This review covers nanostructures, including micelles, rod-like micelles, fibers and peptide beads, self-assembled from de novo designed amphiphilic peptides. The latter are promising candidates for the development of nanoscale carrier systems because they are completely composed of amino acids. In addition to designing primary sequences, secondary structure and external parameters are also discussed with respect to their impact on self-assembly. Moreover, the assembly process itself is examined. Potential applications range from gene and drug delivery devices to diagnostics, thereby highlighting the versatility of the system.
\end{abstract}

Keywords: Amphiphilic peptides · Biomaterial · Bionanotechnology · Gene delivery · Self-assembly

\section{Introduction}

Nature demonstrates an extraordinary variety of self-assembling molecules, such as proteins and nucleic acids. They play a crucial role in all life on earth and are engineered and optimized throughout the stages of evolution as very effective and efficient but complex entities.

Focusing on polypeptides, for instance proteins and enzymes, the building units are amino acids. There are 20 canonical amino acids used in natural biological systems. With the exception of glycine, all are chiral and hence can have two configurations, although generally only the L-configuration is of biological importance. Depending on the sequence, peptides form ordered secondary structures such as $\alpha$-helices or $\beta$-sheets, stabilized by non-covalent interactions such as $\mathrm{H}$-bonding, $\pi$-stacking, and ionic and hydrophobic interactions. Due to the large variety of building units, a nearly endless number of unique peptides can be designed, each with its own three-dimensional conformation and function.

\footnotetext{
${ }^{\star}$ Correspondence: Prof. Dr. W. P. Meier Department of Chemistry

University of Basel

Klingelbergstrasse 80

$\mathrm{CH}-4056$ Basel

E-mail: wolfgang.meier@unibas.ch
}

In past decades, many peptide-based materials have been investigated, the main groups being peptide amphiphiles (PAs) ${ }^{[1,2]}$ and amphiphilic peptides (AP). The latter consist solely of amino acids, in contrast to peptide amphiphiles, which can contain other groups such as alkyl chains, phospholipids and polymers. ${ }^{[3,4]}$ There are several recently published reviews covering the field of PAs. ${ }^{[1,5]}$

The sequence of amphiphilic peptides is organized into two regions: the hydrophobic and the hydrophilic part. The latter is predominantly occupied by charged and polar amino acids; these are: arginine $(\mathrm{R}),{ }^{[6,7]}$ histidine $(\mathrm{H}),{ }^{[7,8]}$ lysine $(\mathrm{K}),{ }^{[9-15]}$ aspartic acid (D), ${ }^{[16,17]}$ glutamic acid (E), ${ }^{[11,18]}$ serine $(\mathrm{S})$, threonine $(\mathrm{T})$, asparagine $(N)$, glutamine $(Q)$, and cysteine $(C){ }^{[13]}$ The design of the hydrophobic part is based on amino acids with neutral and nonpolar side-chains such as glycine $(G),{ }^{[16]}$ alanine (A), ${ }^{[15,19]}$ valine (V), ${ }^{[17,20]}$ leucine (L), ${ }^{[9,17]}$ isoleucine (I), ${ }^{[21]}$ methionine $(\mathrm{M})$, phenylalanine $(\mathrm{F}),{ }^{[22,23]}$ tyrosine $(\mathrm{Y})$, and tryptophan (W). ${ }^{[7,10-14,23]}$ Depending on the hydrophobic to hydrophilic ratio and the sequence, various self-assembled structures can be constructed - as indicated in the associated references for the above amino acids - although the hydrophobicity is moderated by the polar character of the peptide's backbone.

Nanomaterials created from these amphiphiles allow additional tuning towards the required functions and properties by design and functionalization of side-chains and end-groups. Every amino acid has its own benefits; cysteine, for instance, can be used as a connection point for labeling or to covalently attach gold. Mastrobattista's group presented studies of conical-shaped amphiphilic peptides assembling into vesicles with a hydrodynamic radius of about
$60 \mathrm{~nm} \cdot{ }^{[18]}$ Their sequences consisted of Ala, Leu, Val, and Trp for the hydrophobic part, and various lengths of glutamic acid as the hydrophilic part. Gazit et al. reported short aromatic Fmoc-peptides that form spheres, tubes, and fibrils.[24] Zhang's group published work about surfactant-like peptides, which can assemble into nanovesicles and nanofibers. ${ }^{[16]}$

Here we focus only on amphiphilic peptides based on a repetitive tryptophanleucine sequence (W-1), and their recent developments. Exploiting their self-assembly, we have been able to form unique welldefined nano- and microstructures (Fig. 1), depending on the choice and arrangement of building units and the parameters of the assembly process.

\section{Amphiphilic Peptides Based on a Repetitive W-I Sequence}

In our laboratories, a multitude of different de novo designed peptides have been created and investigated. The peptides were synthesized by solid phase peptide synthesis (SPPS) and subsequently purified by RP-HPLC, obtaining products of high purity (95\%). The hydrophobic part is based on gramicidin A, an antibiotic that inserts into lipid membranes. Its sequence and structure was analyzed by Sarges et $a l .{ }^{[25]}$ Hydrophobicity is generated by its helical secondary structure, hiding the peptide's backbone from its surrounding milieu. This knowledge can be adapted to a variety of amphiphilic peptides, as described within the current review. Based on the native sequence, we developed two hydrophobic parts, namely gA (-V-G-A-1-A$\left.\mathrm{v}-\mathrm{V}-\mathrm{v}-[\mathrm{W}-\mathrm{l}]_{3}-\mathrm{W}-\mathrm{NH}_{2}\right)$ and a truncated version gT, containing only the tryptophanleucine repeating unit $\left(-[\mathrm{W}-\mathrm{l}]_{3}-\mathrm{W}-\mathrm{NH}_{2}\right)$. 


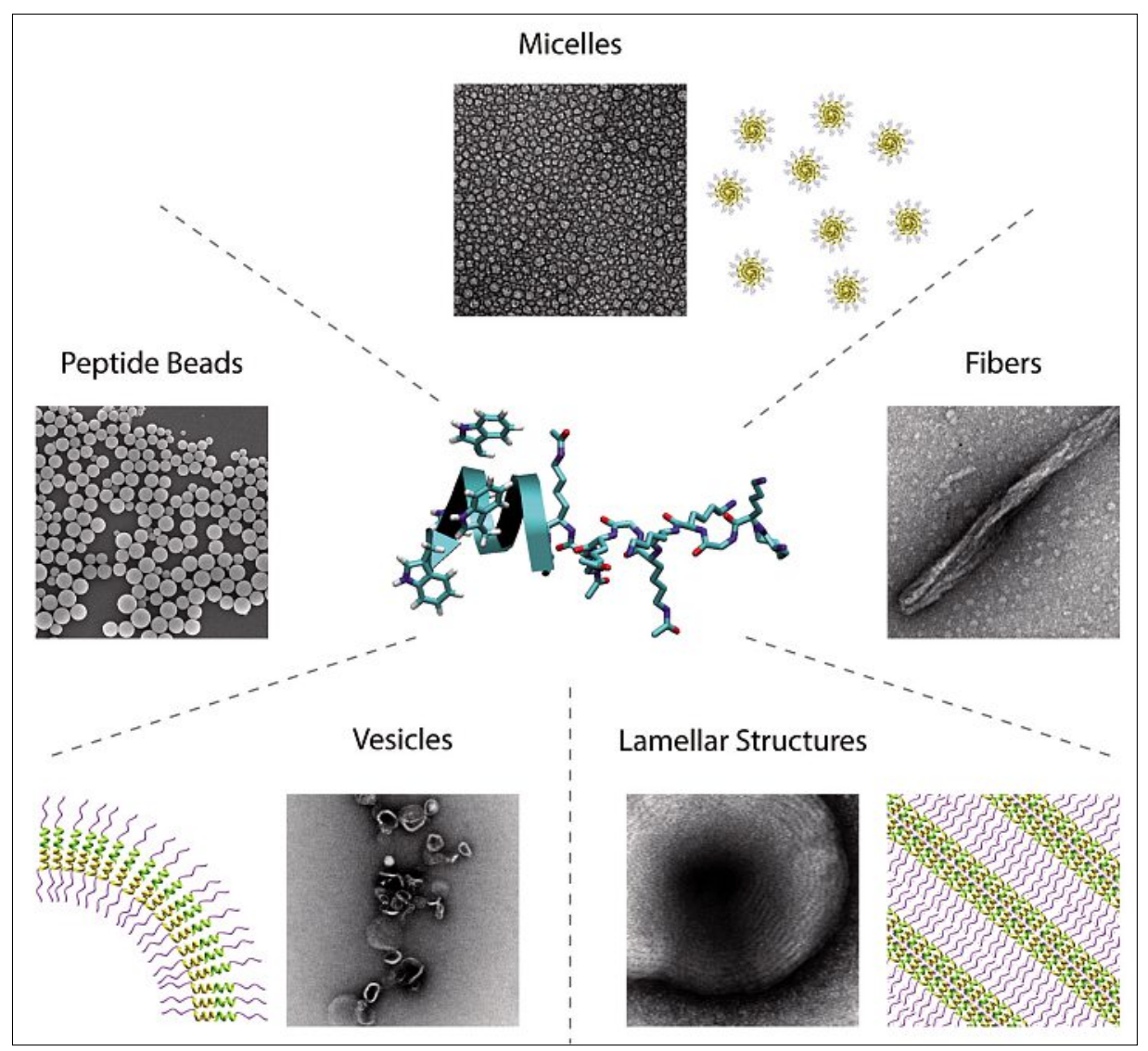

Fig. 1. Overview of self-assembled structures using amphiphilic peptides ranging from micelles, fibers, vesicles and lamellar structures to spherical solid particles termed peptide beads.

Both the gA and gT hydrophobic segments have a C-terminal amide group. Circular dichroism and IR investigations revealed a secondary structure that included a $\beta$-turn motif and $\mathrm{H}$-bonds for the gT hydrophobic part. ${ }^{[14,26]}$ The crucial influence of secondary structure on the peptides' assemblies was shown by replacing D-leucines with $\mathrm{L}-$ leucines, whereby the gT hydrophobic part changed its secondary structure such that amphiphilic nature was no longer present, leading to amorphous assemblies. ${ }^{[14]}$ Charged or polar amino acids served as the hydrophilic part, such as lysine, acetylated lysine (X), cysteine, and/or glutamic acid.

\section{Micelles and Fibers}

Micelles are spherical objects formed by amphiphilic molecules in aqueous solution. This process is driven by an enhanced level of free energy due to the exposure of hydrophobic moieties to water. To overcome this, the molecules begin to orient themselves, hiding hydrophobic parts in a core surrounded by hydrophilic moieties.

$\mathrm{K}_{3}-\mathrm{gT}$ and $\mathrm{C}-\mathrm{K}_{3}-\mathrm{gT}$, oligopeptides with trilysine hydrophilic parts, form micelles with hydrodynamic radii of about $10 \mathrm{~nm}$. They similarly exhibit a surfactant-like behavior, e.g. the critical micelle concentration (CMC) of $\mathrm{C}-\mathrm{K}_{3}-\mathrm{gT}$ is $0.23 \mathrm{mmol}$
$\mathrm{L}^{-1} \cdot{ }^{[12]}$ Decreasing the number of charges by acetylating the lysines [( $\varepsilon$-position, $\mathrm{K}($ acetyl $)=\mathrm{X})$ and the $\mathrm{N}$-terminus (Ac)], lowers the overall hydrophilicity. Acetylated amphiphiles, such as Ac- $\mathrm{X}_{3}-\mathrm{gT}$, AcC- $\mathrm{X}_{3}-\mathrm{gT}$ and Ac- $\mathrm{X}_{3}$-gT-C, form spherical objects in the sub-micrometer range, named peptide beads. The formation process proceeds over micelles, which then aggregate with decreasing ethanol content (see peptide bead section). Aggregation of micelles from $\mathrm{K}_{3}-\mathrm{gT}$ and $\mathrm{C}-\mathrm{K}_{3}-\mathrm{gT}$ can also be achieved by addition of salts.[13]

To understand the impact of changes in the primary sequence, we produced and investigated a collection of ten peptides. We combined a gA hydrophobic part with a hydrophilic one of lysines and acetylated lysines in different ratios. Systematic change in the degree of acetylation (DA) provided control of the number of charges and thereby hydrophilicity. Beginning with $\mathrm{K}_{8}-\mathrm{gA}$, we successively increased the DA to fully acetylated Ac- $\mathrm{X}_{8}-\mathrm{gA}$, comprising no charges and therefore no electrostatic repulsion. Interestingly, between $\mathrm{K}_{2} \mathrm{X}_{6}-\mathrm{gA}$ and $\mathrm{K}_{3} \mathrm{X}_{5}-\mathrm{gA}$ the secondary structure of the hydrophilic part changed dramatically from $\beta$-sheets to $\alpha$-helices, accompanied by different properties. $\mathrm{K}_{2} \mathrm{X}_{6}$-gA self-assembled into fibers with a length ranging from 30 to $800 \mathrm{~nm}$, while $\mathrm{K}_{3} \mathrm{X}_{5}$-gA formed micelles and worm-like micelles. ${ }^{[10]}$
These results concur with recently published studies from Lee et al. ${ }^{[27]}$ According to those studies, peptides with $\beta$-sheet secondary structure preferentially form fibers, due to stabilizing hydrogen bonds perpendicular to the radial ordered unimers. The CMCs were analyzed to be about $0.2 \mathrm{mmol} \mathrm{L}^{-1} \cdot{ }^{[10]}$ An in situ reversible acetylation could convert micelles into fibers and vice versa, a behavior that could be exploited in molecular switches.

The micellar core also provides space for hydrophobic drug encapsulation and can thus be used as a drug delivery system. ${ }^{[28]}$ When modifying the trilysine part of the peptide $\mathrm{K}_{3}$-gT with an additional terminal cysteine for specific functionalization or hybridization, self-assembly behavior remained the same.[13]

\section{Peptide Membranes - Peptosomes and Lamellar Phases}

Nano-compartmentalization, using vesicles, separates the inner medium from its external environment via a membrane of lipids (liposomes), polymers (polymersomes), or peptides (peptosomes). The cavity is appropriate for sheltering fragile biomolecules such as proteins and enzymes, or to lock up soluble toxic payloads. ${ }^{[29]}$ Insoluble molecules can also be accommodated in the hydrophobic membrane. Lipid membranes are omnipresent in nature and serve as a role model in the design of synthetic analogues with improved stability and functionality. ${ }^{[30]}$

To create polymersomes/membranes in our laboratories, we used amphiphilic block-copolymers, including (PMOXA- $b$-PDMS- $b$-PMOXA), ${ }^{[31]}$ (PEG- $b$-PMCL), ${ }^{32]} \quad$ (PEG- $b$-PMCL- $b$ PDMAEMA) ${ }^{[33]}$ and (PB- $b$-PEG). ${ }^{[34]}$ Initially we struggled to extend the applicable self-assembling materials to include amphiphilic peptides. Solely hydrophobic interactions and geometrical aspects were not sufficient to achieve the desired selfassembly into peptosomes. Therefore, we had to modify and improve the original peptide sequence, to remove potential 'barriers/pitfalls' and to implement additional interaction sites.

Ac- $\mathrm{X}_{8}-\mathrm{gA}$ is an amphiphilic peptide that should have the potential to form a vesicular structure. However, it was instead shown to form fibers (see section on Micelles and Fibers). We identified the C-terminal amide functionality, a remainder from the peptide synthesis, as potentially causing difficulties in the formation of an entirely hydrophobic membrane. We therefore masked this group by esterification to yield Ac- $\mathrm{X}_{8}$-gA-OEt. Subsequent analysis of the self-assemblies showed complete absence of fibers and formation 
of vesicles. This result confirmed our initial assumption that improving perpendicular interaction with respect to a membrane would facilitate membrane formation. On the other hand, improving lateral interaction should similarly help in forming a peptidic membrane. We then synthesized gA with a hydrophilic hexa-glutamic acid (E) component, Ac- $\mathrm{E}_{6}-\mathrm{gA}$. The carboxylic group of glutamic acid is able to interact intermolecularly with another carboxylic group and therefore, polyglutamic acids were also found to associate. ${ }^{[35,36]}$ The selfassembled structures of Ac- $\mathrm{E}_{6}-\mathrm{gA}$ were analyzed by SLS, DLS, TEM and CryoTEM, confirming the formation of peptide micelles and vesicles.

Because lipid and polymer vesicles can be used for encapsulation, we also studied the ability of our peptide vesicles to host hydrophilic (Alexa Fluor 488) and hydrophobic payloads (BODIPY 650/665). By including these fluorophores in the formation process, we were able to use laser scanning microscopy (LSM) to observe how they embed within the micellar and vesicular structures. Using $\mathrm{pH}$-sensitive amino acids also provided the system with a responsive behavior that may prove useful in medical or sensor settings. The $\mathrm{pH}$ response is determined by the $\mathrm{pk}_{\mathrm{a}}$ of the amino acids used, which is consistent with the observed precipitation below a $\mathrm{pH}$ of 4 $\left(\approx \mathrm{pk}_{\mathrm{a}}\right.$ of $\left.\mathrm{E}\right)$. The collapse of the structures may be used for $\mathrm{pH}$-triggered release.

As already described in the introduction, the variety within the amino acid alphabet opens a vast playground to control self-assembly. By introducing desired interactions such as $\mathrm{H}$-bonds and charge compensation, highly ordered organizations can be created. ${ }^{[37,38]}$ We synthesized Ac- $\mathrm{K}_{6}-\mathrm{gA}$, a positively charged derivative of Ac- $\mathrm{E}_{6}-\mathrm{gA}$, then mixed both materials together. In a series of TEM images, it was shown that instead of vesicles, multilamellar structures were generated from a molar ratio of 0.3 to 0.8 . The lamellar thickness of $8.3 \pm 1.3 \mathrm{~nm}$ was attributed to a peptide double-layer. ${ }^{[11]}$ Charge compensation between glutamic acid and lysine, combined with hydrophobic interaction, is the apparent driving force towards layer formation.

From a conceptual point of view, peptide membranes are fascinating as analogues to lipid and polymer membranes. However, the low number of publications may illustrate the challenges associated, in particular when using short, amphiphilic oligopeptides. Nevertheless, encapsulation properties of such amino acid-based materials may allow their use as drug and gene delivery vehicles. ${ }^{[39]}$ In this respect, proven polymersomes concepts, such as targeting, may also lead to a fast development of advanced peptosomes. ${ }^{[40,41]}$

\section{Peptide Beads}

Peptide beads are spherical particles in the submicrometer range, formed by self-assembly of the amphiphilic peptide $\mathrm{Ac}-\mathrm{X}_{3}-\mathrm{gT}$ and its close analogues AcC- $\mathrm{X}_{3}-$ gT, AcC(sl)-X -gT, and Ac-X - - TT-C. ${ }^{[13,14]}$ To obtain information about the underlying structure and formation process, we extensively characterized the beads by microscopic and light scattering (LS) techniques, such as AFM, TEM, SEM, SLS and DLS. In summary, the beads exhibited a particle scattering factor and a $\rho$-parameter $\left(R_{\mathrm{g}} / \mathrm{R}_{\mathrm{h}}\right)$ that confirm the beads to be solid spheres. ${ }^{[42]}$ Their density was determined to be about $1.4 \mathrm{~g} \mathrm{~cm}^{-3}{ }^{[13,14]}$ Their size was dependent on the initial peptide concentration, ranging from a radius of 150 to $1500 \mathrm{~nm}$. SEM micrographs often showed round indentations on the bead surfaces, most probably a drying effect due to the last remaining water leaving the particles. All these results fit well together and represent a consistent image of the particles, but give very little information about the self-assembly process itself or the underlying structure.

To better assess these areas, we looked closer into the bead formation process. Peptidic material is usually dissolved in ethanol (EtOH) and dialyzed against double distilled water $\left(\mathrm{dd}_{2} \mathrm{O}\right)$. Therefore we stopped the formation process at different stages, i.e. at concentrations of 40, 20 and $4 \mathrm{wt} \% \mathrm{EtOH}$, and subsequently analyzed by LS and electron paramagnetic resonance (EPR). The resulting fast rotational correlation time indicated predominance of a mobile species at $40 \mathrm{wt} \% \mathrm{EtOH}$ and high temperatures. At lower ethanol content and temperature, a second 'immobile' species emerged as dominant, characterized by a slow rotational correlation time. ${ }^{[13]} \mathrm{LS}$ data for those stages support the EPR results, suggesting that the self-assembly process
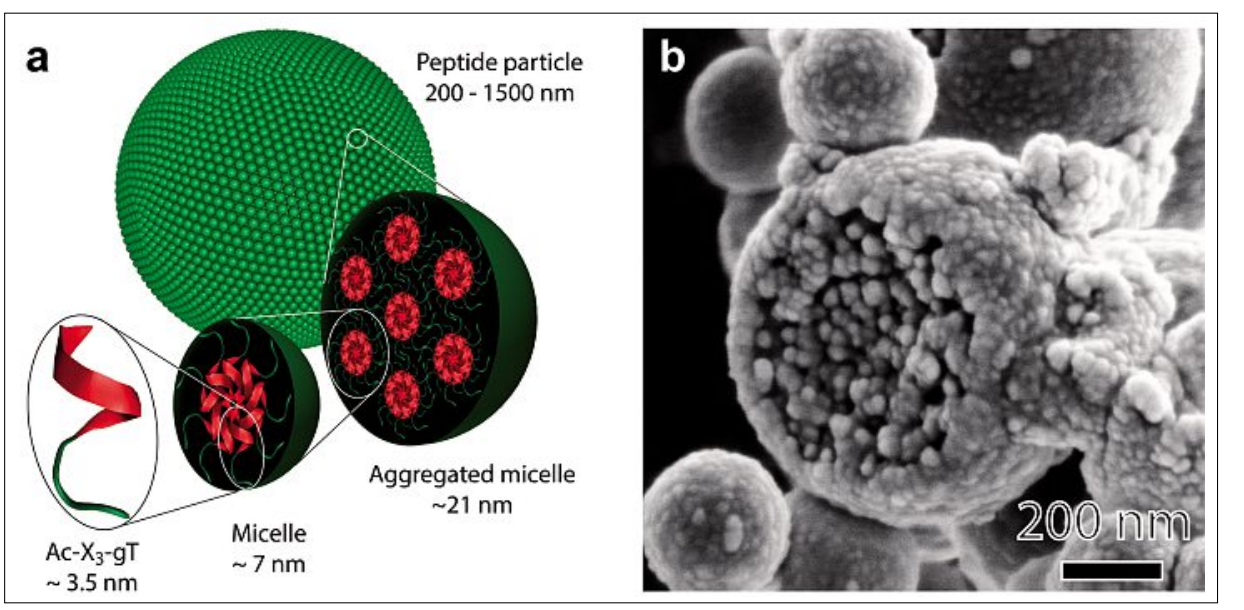

Fig. 2. a) Hierarchical self-assembly of $A c-X_{3}-g T$ into multicompartment micelles termed peptide beads. b) SEM micrograph of an $\mathrm{Ac}-\mathrm{X}_{3}-\mathrm{gT}-\mathrm{C}$ peptide bead, revealing an insight into the inner

structure.

starts in the dissolved state $(\mathrm{EtOH})$. With decreasing EtOH content several stages are passed through: the formation of micelles, their loose aggregation and finally their consolidation in entirely aqueous solution. This aggregated micelle structure can be described as a multi-compartment micelle (MCM) (Fig. 2).

This approach leaves some open questions: Is there a reorganization of the micelles within the peptide beads? Are the micelles still intact? Is there a different substructure present in the peptide beads, which may not have been detected by the EPR experiment? However, further evidence supporting a hierarchical organization of micelle-like substructures came from a combination of electron and atomic force microscopy, and small angle X-ray scattering (SAXS) on Ac- $\mathrm{X}_{3}$-gT beads. ${ }^{[12]}$

Two structure models have been proposed: large compound micelles and an aggregated micelle model (MCM). Based on the available data, it was ultimately impossible to assign a model with reasonable certainty. Both models provide segregated hydrophobic and hydrophilic nanocompartments, due to the micellar substructure and intermicellar regions, respectively. In both models, it is thus possible to embed different payloads with different or/and multiple affinities. At present, the list of embedded payloads includes rose bengal (RB), 5-carboxy-fluorescein, BODIPY 630, Alexa 488, doxorubicin, paclitaxel, siRNA, plasmid DNA and even $8 \mathrm{~nm}$ gold-nanoparticles (Au-NP). ${ }^{[14,26,43]}$ Interestingly, it was observed that the payload is enriched within the peptide beads, such as a 900-fold increase in RB, and RNA/DNA almost completely incorporated. ${ }^{[14,43]}$ Therefore theory and experimental findings are consistent, i.e. the peptide beads provide a matrix in which payloads preferentially dissolve with increasing dielectric constant (dd $\mathrm{H}_{2} \mathrm{O}$ content). 
Polymeric multi-compartment micelles exhibit outstanding properties and are therefore attractive for a wide variety of applications in nanotechnology. [44-46] Similarly our peptidic analogues provide new opportunities, such as acting as a template for ordered Au-NPs. Therefore, we elongated the peptide $\mathrm{C}$-terminus with a cysteine (Ac- $\mathrm{X}_{3}-\mathrm{gT}$-C) for a covalent linkage to $\mathrm{Au}-\mathrm{NPs}$, to introduce them within the hydrophobic micellar cores of the beads. ${ }^{[26]}$ TEM images confirmed ordered $\mathrm{Au}-\mathrm{NP}$ insertion and formation of new composite peptide-gold nanoparticles (CP-Au-NPs). The results underlined the structural features of the peptide beads but also illustrated loading capacity, selfassembling ability and usage as a template. Moreover, their application as a nanocontainer for advanced drug delivery is envisaged, supported by the list of embedded payloads mentioned above.

Recently, we studied peptide beads as a new biocompatible gene and drug delivery tool. First, we illustrated the internalization of the peptide beads into THP-1 macrophages, THP-1 monocytes and hepatocellular carcinoma cells (Huh7). We successfully co-embedded the hydrophilic and hydrophobic anticancer drugs doxorubicin and paclitaxel, which were internalized by THP- 1 monocytes. ${ }^{[43]}$ This led to decreased cell viability, due to the activity of the embedded drugs.

Far more challenging is the delivery of siRNA and plasmid DNA (pDNA), due to a number of barriers including large dimensions of pDNA, fast degradation/instability (DNase) and the need to reach the nucleus (pDNA). ${ }^{[47]}$ Peptide beads were studied regarding this application. Plasmid DNA carrying antibiotic resistance and encoding for shRNA for the $\alpha$ isoform of the catalytic subunit of human PP2A (PP2Ac $\alpha$ ) was embedded and applied to Huh7 cells. ${ }^{[43]}$ Although initial quantitative reverse transcriptase polymerase chain reaction analysis demonstrated only limited silencing, cells that survived culturing in antibioticcontaining media for over three weeks showed significant effect $(\sim 33 \%)$. This system exhibited various advantages, such as high encapsulation efficiency, and thus little effort was needed for post-embedding purification. In summary, proof of concept of our peptide system as a potential gene delivery device was demonstrated. Initial low level of gene silencing might indicate a problem of sufficient and concerted drug release. To overcome this limitation, we currently work on an improved prototype exhibiting a triggered release.

\section{Conclusion}

Derived from gramicidin A, the crucial feature of our amphiphilic peptides is its hydrophobic part, gA and gT. In combination with different hydrophilic segments, in terms of choice and arrangement of amino acids as well as overall length, a variety of self-assembled structures were generated, starting with micelles and fibers, moving to vesicles, and finally to solid spheres. The structures presented are intrinsically very interesting and additionally important for their potential application in medical and diagnostic applications. In particular, we found the amphiphiles easy to synthesis, facile to modify, and robust in their particle formation. The peptide beads are a versatile structure in terms of payload embedding, enrichment of dyes and drugs, i.e. high loading capacity, particle stability, and internalization in different cell lines. Application as a drug and gene delivery tool showed very promising results and optimization efforts are already underway.

\section{Acknowledgment}

Financial support by Gerbert Rüf Stiftung 'Peptide Targeted Transport System' (GRS - 048/11), the SNSF, and the NCCR Nanosciences is gratefully acknowledged. The authors thank Dr. Dirk de Bruyn Ouboter, Prof. Cornelia Palivan and RM Subasic for discussion and reading the manuscript. We also thank Ruth Pfalzberger for her help with the preparation of the graphics.

Received: September 12, 2013

[1] H. Cui, M. J. Webber, S. I. Stupp, Biopolymers 2010, $94,1$.

[2] J. Y. Shu, B. Panganiban, T. Xu, Annu. Rev. Phys. Chem. 2013, 64, 631.

[3] S. Cavalli, F. Albericio, A. Kros, Chem. Soc. Rev. 2010, 39, 241.

[4] I. W. Hamley, Soft Matter 2011, 7, 4122.

[5] R. V. Ulijn, A. M. Smith, Chem. Soc. Rev. 2008, 37, 664.

[6] U. Khoe, Y. Yang, S. Zhang, Langmuir 2009, $25,4111$.

[7] W. Y. Seow, Y.-Y. Yang, Adv. Mater. (Weinheim, Ger.) 2009, 21, 86 .

[8] Q. Tang, B. Cao, H. Wu, G. Cheng, Langmuir 2012, 28, 16126.

[9] V. Breedveld, A. P. Nowak, J. Sato, T. J. Deming, D. J. Pine, Macromolecules 2004, 37, 3943.

[10] T. B. Schuster, D. de Bruyn Ouboter, C. G. Palivan, W. Meier, Langmuir 2011, 27, 4578 .

[11] T. B. Schuster, D. de Bruyn Ouboter, N. Bruns, W. Meier, Small 2011, 7, 2158.

[12] D. de Bruyn Ouboter, T. B. Schuster, A. Mantion, W. Meier, J. Phys. Chem. C 2011, $115,14583$.

[13] T. B. Schuster, D. de Bruyn Ouboter, E. Bordignon, G. Jeschke, W. Meier, Soft Matter 2010, 6, 5596.

[14] C. Dittrich, W. Meier, Macromol. Biosci. 2010, 10, 1406.

[15] C. Chen, F. Pan, S. Zhang, J. Hu, M. Cao, J. Wang, H. Xu, X. Zhao, J. R. Lu, Biomacromolecules 2010, 11, 402.

[16] S. Santoso, W. Hwang, H. Hartman, S. Zhang, Nano Lett. 2002, 2, 687.

[17] S. Vauthey, S. Santoso, H. Gong, N. Watson, S. Zhang, Proc. Natl. Acad. Sci. USA 2002, 99 , 5355.

[18] A. J. van Hell, C. I. C. A. Costa, F. M. Flesch, M. Sutter, W. Jiskoot, D. J. A. Crommelin, W. E.
Hennink, E. Mastrobattista, Biomacromolecules 2007, 8, 2753.

[19] S. Bucak, C. Cenker, I. Nasir, U. Olsson, M. Zackrisson, Langmuir 2009, 25, 4262.

[20] D. J. Adams, K. Holtzmann, C. Schneider, M. F. Butler, Langmuir 2007, 23, 12729.

[21] M. K. Baumann, M. Textor, E. Reimhult, Langmuir 2008, 24, 7645 .

[22] Z. Huang, S. Guan, Y. Wang, G. Shi, L. Cao, Y. Gao, Z. Dong, J. Xu, Q. Luo, J. Liu, J. Mater. Chem. B 2013, 1, 2297.

[23] M. C. Morris, E. Gros, G. Aldrian-Herrada, M. Choob, J. Archdeacon, F. Heitz, G. Divita, Nucleic Acids Res. 2007, 35, e49.

[24] R. Orbach, L. Adler-Abramovich, S. Zigerson, I. Mironi-Harpaz, D. Seliktar, E. Gazit, Biomacromolecules 2009, 10, 2646.

[25] R. Sarges, B. Witkop, J. Am. Chem. Soc. 1965, 2027.

[26] D. De Bruyn Ouboter, T. B. Schuster, S. J. Sigg, W. Meier, Colloids Surf., B 2013, 112, 542.

[27] O.-S. Lee, S. I. Stupp, G. C. Schatz, J. Am. Chem. Soc. 2011, 133, 3677.

[28] A. Ponta, Y. Bae, Pharm. Res. 2010, 27, 2330.

[29] A. Najer, D. Wu, D. Vasquez, C. G. Palivan, W. Meier, Nanomedicine 2013, 8, 425.

[30] X. Zhang, P. Tanner, A. Graff, C. G. Palivan, W. Meier, J. Polym. Sci. Part A: Polym. Chem. 2012, 50, 2293.

[31] K. Langowska, C. G. Palivan, W. Meier, Chem. Commun. 2013, 49, 128.

[32] J. Braun, N. Bruns, T. Pfohl, W. Meier, Macromol. Chem. Phys. 2011, 212, 1245.

[33] Y. Matter, R. Enea, O. Casse, C. C. Lee, J. Baryza, W. Meier, Macromol. Chem. Phys. 2011, 212, 937.

[34] S. Belegrinou, J. Dorn, M. Kreiter, K. KitaTokarczyk, E.-K. Sinner, W. Meier, Soft Matter 2009, 6, 179.

[35] T. Makovec, Biochem. Mol. Biol. Educ. 2000, 28, 244.

[36] E. J. Spek, Y. Gong, N. R. Kallenbach, J. Am. Chem. Soc. 1995, 117, 10773.

[37] E. Jahnke, I. Lieberwirth, N. Severin, J. P. Rabe, H. Frauenrath, Angew. Chem., Int. Ed. 2006, 45, 5383.

[38] G. W. M. Vandermeulen, H.-A. Klok, Macromol. Biosci. 2004, 4, 383.

[39] M. de Raad, E. A. Teunissen, D. Lelieveld, D. A. Egan, E. Mastrobattista, J. Controlled Release 2012, 158, 433.

[40] M. Randal, A. A. Kossiakoff, Protein Sci. 1998, 7, 1057.

[41] H. Ringsdorf, J. Polym. Sci.: Polym. Symp. 1975, 51, 135.

[42] S. Egli, H. Schlaad, N. Bruns, W. Meier, Polymers 2011, 3, 252.

[43] D. De Bruyn Ouboter, T. B. Schuster, V. Shanker, M. Heim, W. Meier, J. Biomed. Mater. Res., Part A 2013, doi: 10.1002/jbm.a.34778.

[44] H. von Berlepsch, C. Boettcher, K. Skrabania, A. Laschewsky, Chem. Commun. 2009, 2290.

[45] J.-F. Lutz, A. Laschewsky, Macromol. Chem. Phys. 2005, 206, 813.

[46] S. Kubowicz, J.-F. Baussard, J.-F. Lutz, A. F. Thuenemann, H. von Berlepsch, A. Laschewsky, Angew. Chem., Int. Ed. 2005, 44, 5262.

[47] K. Nakanishi, T. Sakiyama, Y. Kumada, K. Imamura, H. Imanaka, Curr. Proteomics 2008, 5,161 . 